\title{
Mantenimiento Correctivo Aplicado a un Sitio Basado en Joomla. Una Propuesta Centrada en la Accesibilidad
}

\author{
Daiana E. Casaro, Pedro L. Alfonzo, Sonia I. Mariño, María V. Godoy \\ Departamento de Informática. Facultad de Ciencias Exactas y Naturales y Agrimensura. 9 de Julio No 1449 . 3400. Corrientes. \\ Argentina. Universidad Nacional del Nordeste. \\ plalfonzo@hotmail.com,simarinio@yahoo.com,mvgg2001@yahoo.com
}

\begin{abstract}
Resumen-Se presenta una experiencia de personalización de un sitio basado en un sistema gestor de contenidos de libre distribución o CMS, atendiendo su amplia aplicabilidad en la Industria del Software y el Sector de Servicios Informáticos. Dado que un CMS puede ser mejorado por la comunidad de desarrolladores, se considera al mantenimiento correctivo como parte integral del ciclo de vida de desarrollo del software. En este trabajo, se abordan los estándares propuestos por el Consorcio W3C que permiten el acceso a los contenidos web y la verificación del cumplimiento de las pautas WCAG 2.0, durante el proceso de desarrollo del sitio web.
\end{abstract}

Palabras Claves - Accesibilidad Web, mantenimiento correctivo, gestor de contenidos.

\section{INTRODUCCIÓN}

La Accesibilidad Web (AW) referencia el acceso universal a este servicio de Internet, independientemente del tipo de hardware, software, infraestructura de red, idioma, cultura, localización geográfica y capacidades de los usuarios [1]. La Industria del Software y el Sector de Servicios Informáticos (SSI) han cobrado gran connotación en los últimos tiempos, dado el crecimiento de las TIC en diversos aspectos del mercado y la sociedad. Los sitios generados para apoyar a las organizaciones que ofrecen servicios a través de la web, adquieren importancia al incrementarse la cantidad de internautas, sin embargo en numerosos sitios web existen barreras que dificultan el acceso masivo a sus contenidos.

En octubre de 2012, el Consorcio W3C difundió la aprobación de las Pautas de Accesibilidad al Contenido Web (WCAG) 2.0 como un estándar internacional ISO/IEC (ISO / IEC 40500:2012). Siguiendo estas pautas harán el contenido accesible a una gama más amplia de personas con discapacidad, incluyendo ceguera y baja visión, sordera y pérdida de la audición, problemas de aprendizaje, limitaciones cognitivas, limitado movimiento, entre otros [2].

La WCAG 2.0 se organiza en torno a cuatros principios teóricos que buscan garantizar el acceso a los contenidos. A continuación se sintetizan los principios y pautas de la WCAG $2.0[4]$ :

Principio 1. Perceptible: La información y los componentes de la interfaz de usuario deben ser mostrados a los usuarios en formas que ellos puedan entender.

- Pauta 1.1: Texto alternativo: Proporciona texto alternativo para el contenido que no sea textual, así podrá ser transformado en otros formatos que la gente necesite, como caracteres grandes, lenguaje braille, lenguaje oral, símbolos o lenguaje más simple.
- Pauta 1.2: Contenido multimedia dependiente del tiempo: Proporcione alternativas sincronizadas para contenidos multimedia sincronizados dependientes del tiempo.

- Pauta 1.3: Adaptable: Crear contenido que pueda ser presentado de diferentes formas sin perder ni información ni estructura.

- Pauta 1.4: Distinguible: Facilitar a los usuarios ver y escuchar el contenido incluyendo la distinción entre lo más y menos importante.

Principio 2. Operable: Los componentes de la interfaz de usuario y la navegación deben ser manejables.

- Pauta 2.1: Teclado accesible: Poder controlar todas las funciones desde el teclado.

- Pauta 2.2: Tiempo suficiente: Proporciona tiempo suficiente a los usuarios para leer y utilizar el contenido.

- Pauta 2.3: Ataques epilépticos: No diseñar contenido que pueda causar ataques epilépticos.

- Pauta 2.4: Navegación: Proporciona formas para ayudar a los usuarios a navegar, a buscar contenido y a determinar dónde están estos.

Principio 3. Comprensible: La información y las operaciones de usuarios deben ser comprensibles.

- Pauta 3.1. Legible. Hacer el contenido de texto legible y comprensible.

- Pauta 3.2. Previsible. Hacer la apariencia y la forma de utilizar las páginas web previsible.

- Pauta 3.3. Entrada de datos asistida. Ayudar a los usuarios a evitar y corregir errores.

Principio 4. Robusto: El contenido debe ser los suficientemente robustos como para ser interpretado de forma fiable por una variedad de aplicaciones de usuarios, incluyendo las ayudas técnicas.

- Pauta 4.1. Compatible. Maximizar la compatibilidad con las aplicaciones de usuarios actuales y futuras, incluyendo las ayudas técnicas.

Por otra parte, la Ingeniería del Software (IS) define métodos y herramientas orientados a la construcción disciplinada de artefactos software y potenciar el SSI. La AW, desde la IS, se refiere a cómo se debe codificar y presentar la información cuando se diseña un sitio para lograr que las personas con o sin alguna discapacidad puedan percibir, entender, navegar e interactuar de forma efectiva con la $\mathrm{Web}$, así como también crear y aportar contenido.

Otro aspecto de relevancia en la IS es el mantenimiento de sus productos a fin de lograr una mayor funcionalidad de los mismos en la comunidad de usuarios.

Se considera al mantenimiento del software (MS) como "la modificación de un producto software después de la entrega para corregir fallos, para mejorar el rendimiento $\mathrm{u}$ otros 
atributos, o para adaptar el producto a un entorno modificado" [9]. Esta definición indica que las actividades de mantenimiento comienzan después que el producto está en funcionamiento. En ocasiones, algunas actividades pueden comenzar antes de la entrega del producto.

Para [8] el proceso de desarrollo continúa cuando un sistema es entregado durante su tiempo de vida. Los sistemas desarrollados sufren cambios para su permanencia y utilidad. Una vez que el software comienza a emplearse, surgen nuevos requisitos y los existentes cambian. Algunas partes del mismo tienen que modificarse para corregir errores detectados en su funcionamiento, adaptarlo a una nueva plataforma, mejorar su rendimiento, entre otras características no funcionales.

En este sentido [8] distingue tres tipos de mantenimiento: i) Mantenimiento correctivo: realizar cambios en el software para reparar defectos; ii) Mantenimiento adaptativo: modificar el software para adaptarlo a un nuevo entorno o nuevos requerimientos; iii) Mantenimiento perfectivo: perfeccionar el software implementando nuevos requerimientos $\mathrm{o}$ bien, mantener la funcionalidad del sistema pero mejorando su estructura y su rendimiento.

Se coincide con [10] en que los Sistemas Gestores de Contenidos o CMS son ampliamente aplicados en una diversidad de soluciones para mediar la comunicación con los usuarios finales. Además de existir numerosas herramientas comprendidas en esta categoría del software de libre distribución ampliamente instalada en la comunidad de programadores, como ser Joomla [11], Drupal [12], Moodle [13], entre otros; así como diversas experiencias exitosas de su utilización en diversos dominios del conocimiento.

En este trabajo se considera relevante el estudio y difusión de contenidos referentes a la accesibilidad a los contenidos web; y se expone una experiencia de personalización de un sitio basado en un sistema gestor de contenidos de libre distribución o CMS como es Joomla [11], dada su amplia aplicabilidad en el SSI. Además, un CMS puede ser mejorado por la comunidad de desarrolladores, en este sentido, se considera al mantenimiento correctivo como parte integral del ciclo de vida de desarrollo del software, a los efectos que el producto software elaborado sea operativo por un mayor número de usuarios finales, maximizando las inversiones de las organizaciones.

\section{METODOLOGÍA}

Se sintetiza la metodología aplicada en el diseño y construcción de un Sitio Web de difusión de contenidos orientado a aplicar mantenimiento correctivo a un CMS de libre distribución.

Etapa 1. Selección de CMS de libre distribución.

Etapa 2. Desarrollo de un prototipo de CMS de difusión de información.

- Análisis y diseño de interfaces. Definición de perfiles de usuarios para establecer secciones privadas y públicas del sitio web.

- Codificación y desarrollo. Instalación y configuración de la plantilla base e incorporación de módulos orientados al registro de usuarios y generación de contactos con los potenciales visitantes, opciones del menú principal, ruta y sitios de interés.

Etapa 3. Etapa de pruebas centrada en el cumplimiento de criterios o las Pautas de Accesibilidad al Contenido (WCAG 2.0) como requerimiento no funcional al prototipo de sitio web desarrollado. En este sentido se aplicó mantenimiento correctivo a los módulos propuesto por Joomla a fin de mejorar la accesibilidad del sitio.

Etapa 4. Aplicación del mantenimiento correctivo centrado en la accesibilidad.

Etapa 5. Difusión de los resultados.

\section{RESULTADOS}

En esta sección se presentan los resultados del mantenimiento correctivo aplicado a un prototipo de Sitio Web para la difusión de contenidos. A fin de validar la propuesta se desarrolló un entorno virtual con 7 secciones o páginas como se resume en la Tabla 1.

\section{A. Experiencias de mantenimiento correctivo.}

Se sintetizan los resultados obtenidos derivados de la aplicación de la etapa 2 expuesta en la metodología.

- Análisis y diseño de interfaces

En esta etapa se realizó un estudio preliminar obteniendo información necesaria para la definición del problema. También se determinaron los requerimientos del sistema evaluando alternativas.

Los perfiles de usuarios a los cuales está dirigidos los productos de software determinan el diseño del sistema informático. La interfaz del usuario es donde se presentan los problemas de accesibilidad e impide a personas con alguna discapacidad alcanzar su objetivo cuando navega un sitio Web [3].

El uso de estándares en etapas tempranas del proceso de construcción de Sitios Web, representa una manera de iniciar un proyecto innovador de desarrollo de software de calidad [5]. Lo expuesto se traduce en la generación de productos de calidad, especialmente por el uso masivo de software desde Internet. En este sentido, se integró la accesibilidad como un atributo de calidad durante el proceso de desarrollo, siendo este uno de los factores relevantes en la calidad de software y aplicada en la elaboración del sitio Web de difusión.

- Codificación y desarrollo

La generación de código se refiere a la construcción de los ambientes virtuales como aquellas tareas referidas a añadir comportamiento a estos ambientes.

Se realizaron tareas de instalación y configuración de la plantilla, módulos y componentes, considerando los estándares requeridos por el CMS Joomla 3.2.3. y su API. Instalado el CMS, a través del 'Gestor de Plantillas (Fig. 1), se procedió a la elección e instalación de una plantilla base. En la Fig. 2, se observa la plantilla seleccionada, la cual fue modificada de acuerdo a lo explicitado en la etapa de análisis y se estableció como predeterminada. Disponible la plantilla base, se procedió a la incorporación de componentes básicos necesarios para proporcionar funcionalidad al sitio, como ser: menú principal, ruta, formulario de acceso, acceso privado y sitios de interés. La Fig. 3 ilustra las opciones incluidas en el menú principal del Sitio Web de difusión desde el 'Gestor de Menús' de Joomla y disponible para los usuarios explicitados anteriormente (registrados o no).

B. Pruebas centradas en la verificación del cumplimiento WCAG 2.0.

Se realizaron las pruebas del sitio desarrollado. Las validaciones de accesibilidad durante el proceso de gestión de contenidos son validaciones preventivas con el objetivo de la detección temprana de problemas de accesibilidad, previas a la 


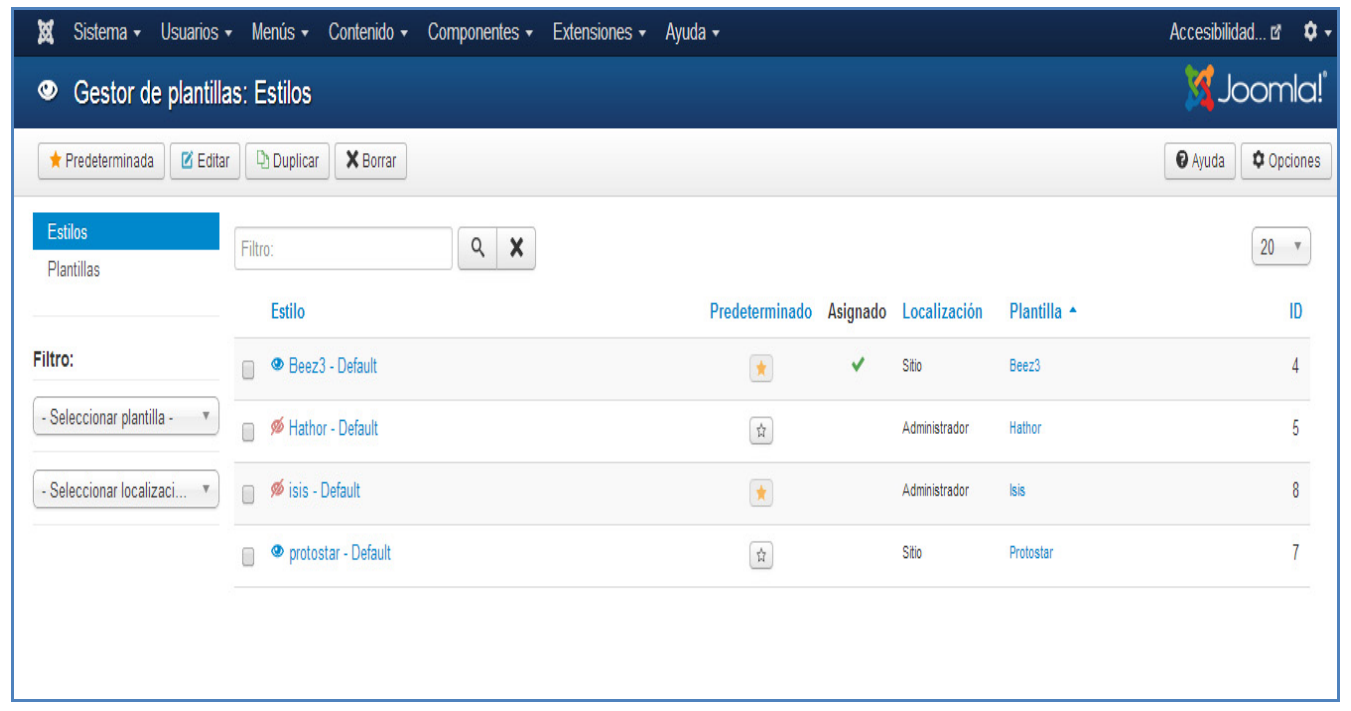

Fig. 1. Selección de la plantilla base desde el Gestor de plantillas del CMS.

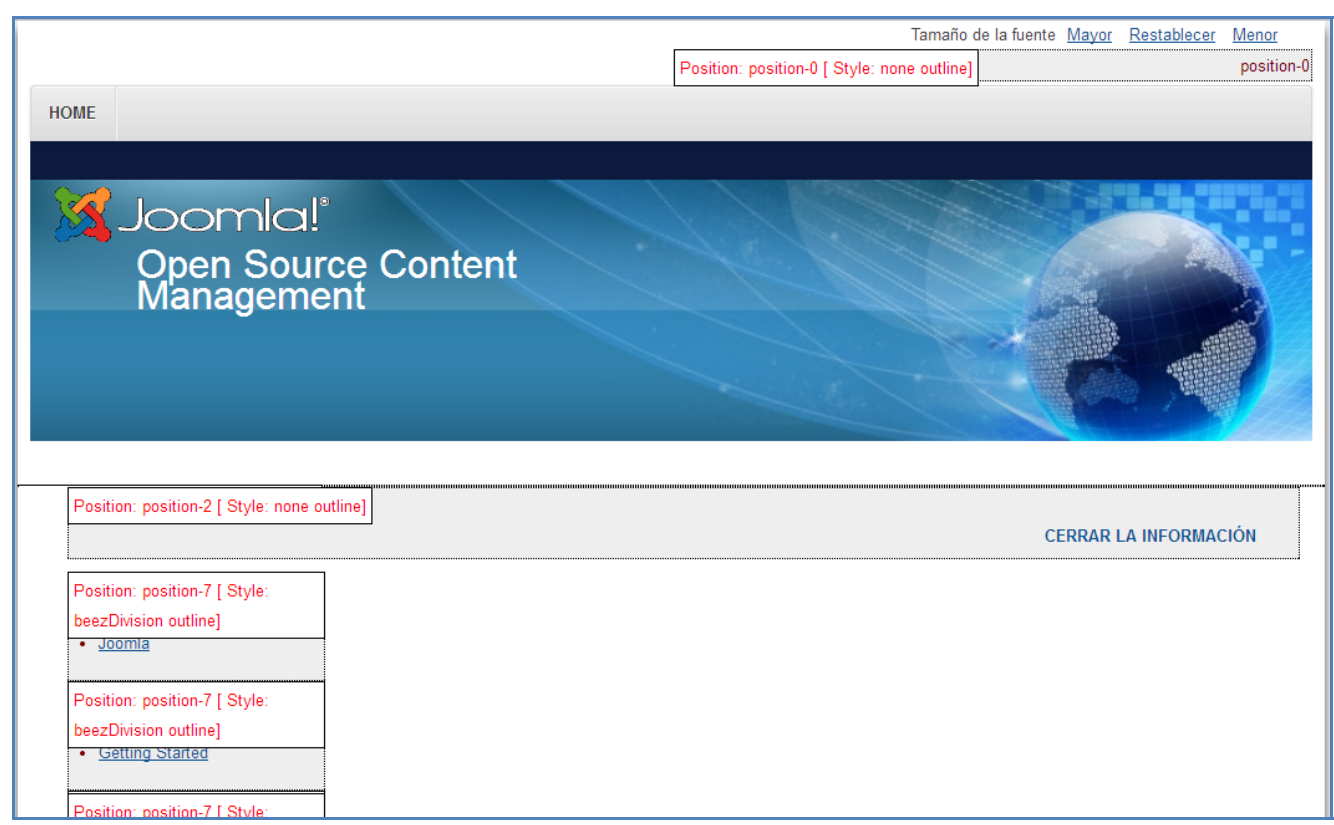

Fig. 2. Interfaz de la plantilla base elegida.

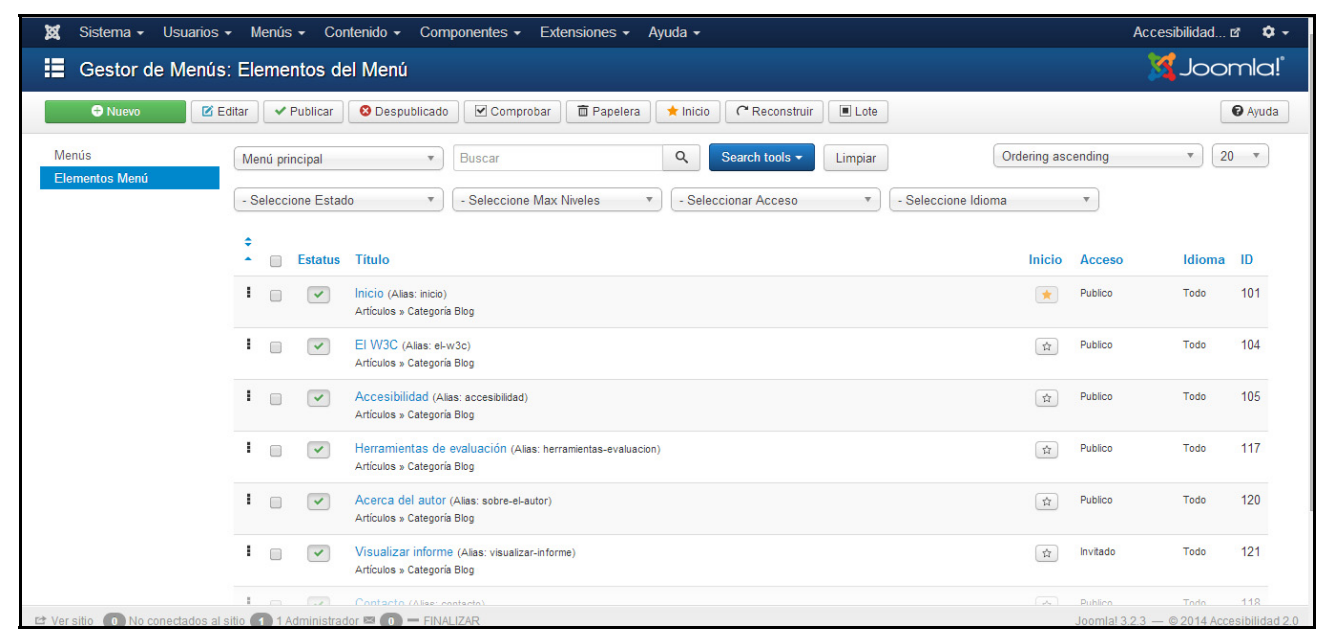

Fig. 3. Panel de configuración del menú principal del Sitio Web. 
publicación de los contenidos en el sitio, es decir, se evidencia la aplicación de mantenimiento correctivo.

Para proceder a la revisión de la accesibilidad a través de los validadores automáticos, se implementó el sitio. Se verificó la accesibilidad con el validador automático TAW [6] en el "Nivel AA" de la WCAG 2.0, para constatar el cumplimiento de los principios establecidos. Además, se utilizó el navegador Mozilla Firefox con su complemento Web Developer Extensión [7], para la evaluación manual de los puntos recomendados por los validadores automáticos.

\section{Aplicación del mantenimiento correctivo centrado en la accesibilidad.}

Incluye los procesos de control, mejora y optimización, teniendo en cuenta la accesibilidad a los contenidos de acuerdo al explicitado por la W3C, en los componentes incorporados, lo que trae como consecuencia la reconfiguración de algunos de los módulos. En este sentido los componentes agregados como los formularios de registro de usuario y de contacto, fueron reutilizados e incluyó modificación del código (Fig. 4 y Fig. 5), para adaptarlos a los requerimientos.

En la Fig. 6 se puede observar el código CSS (hojas de estilos en cascada) para el diseño del sitio Web generado por el gestor de contenido; y en la Fig. 7, se visualiza el código con las correcciones realizadas.

\section{Medición de la accesibilidad como un aspecto del} mantenimiento correctivo.

En el Sitio Web se dispone de un panel frontal (Front-end), medio de interacción con los usuarios registrados y no registrados que acceden a la información. Se estableció como Requerimiento no funcional: ser accesible a la comunidad en general. Por lo expuesto se evaluaron las páginas que lo componen de acuerdo al nivel AA de las WCAG 2.0. En la Tabla 1 se presentan los resultados obtenidos al evaluar con el validador TAW [6], observándose la carencia de errores automáticos según los principios y sus pautas previamente mencionados.

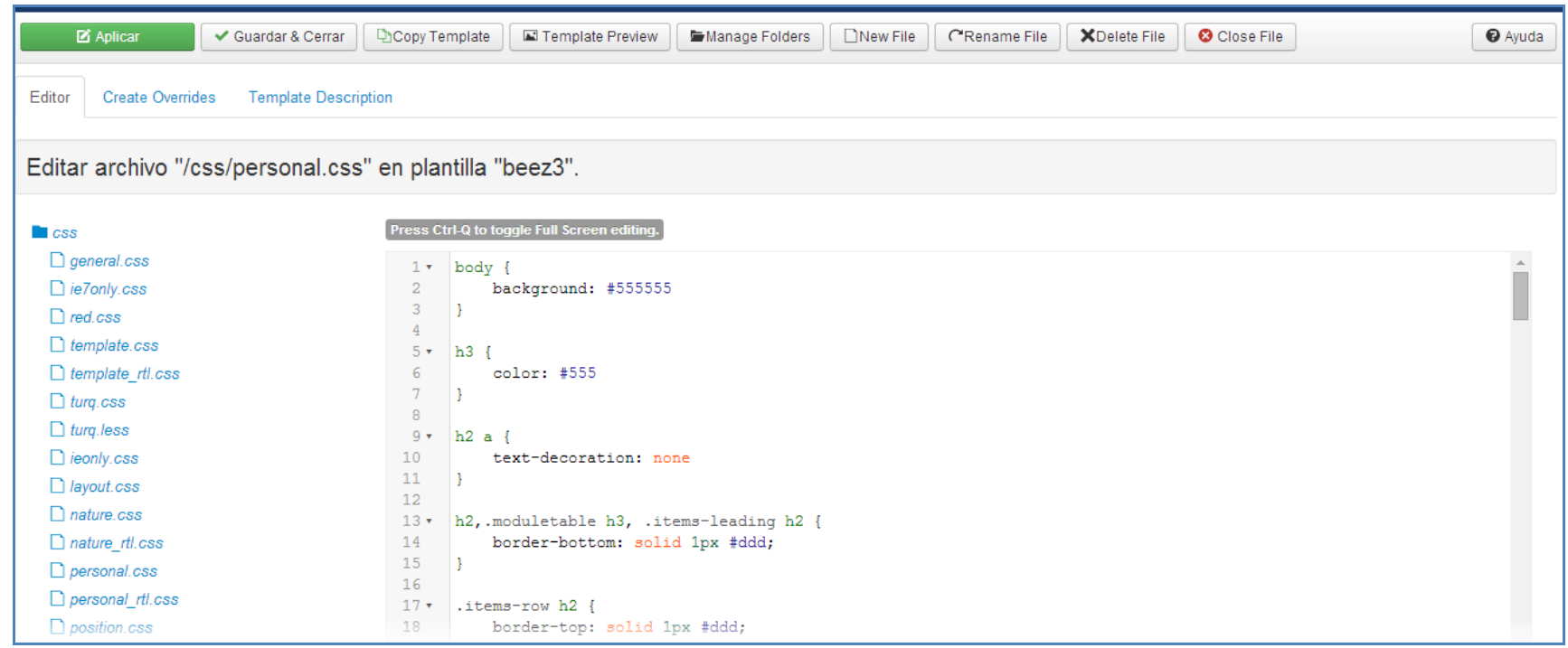

Fig. 6. Visualización del código de la CSS generado por el CMS.

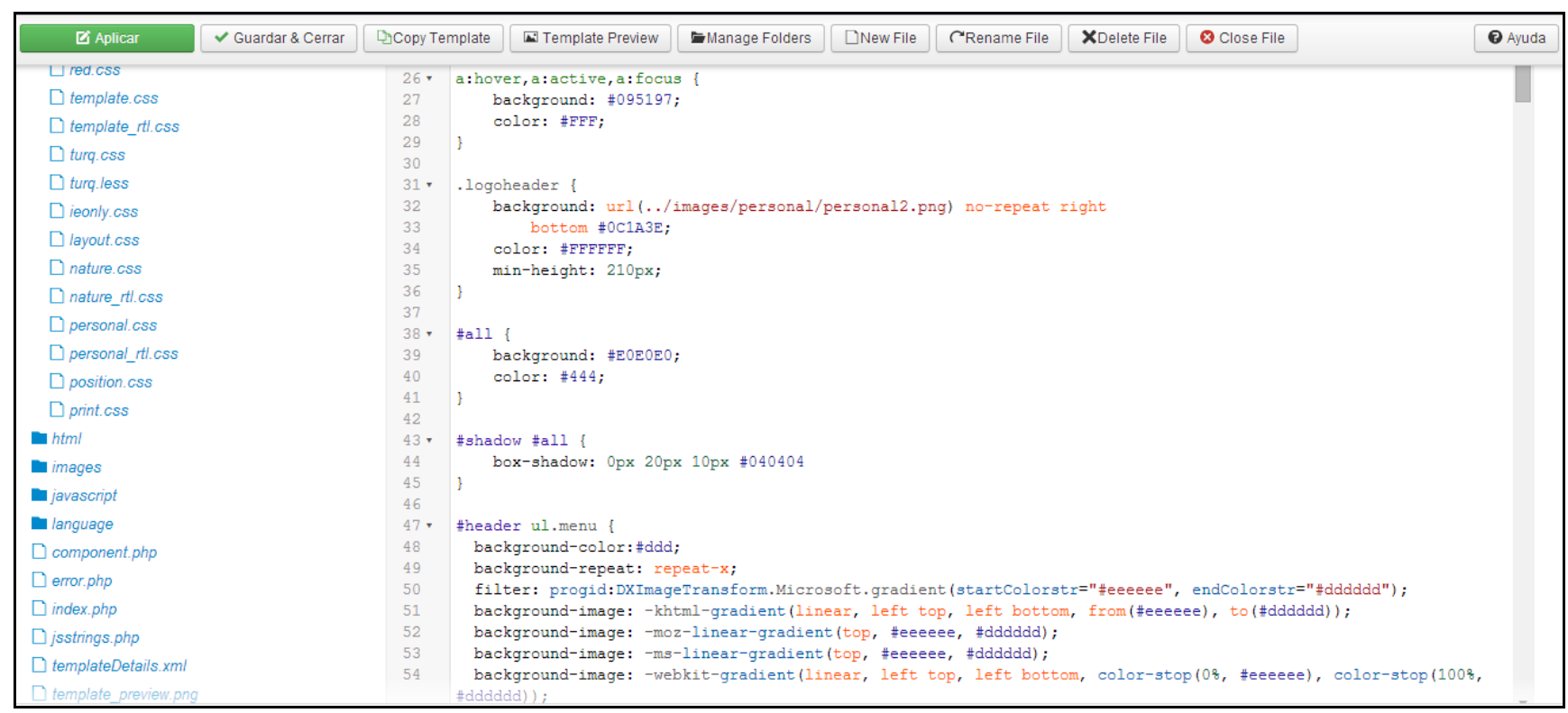

Fig. 7. Visualización de la modificación del código realizada a la CSS. 
La mayoría de los revisores clasifican los errores de accesibilidad en dos tipos:

- Errores automáticos: fueron detectados por la herramienta con seguridad. La alternativa de solución consiste en modificar el código del sitio.

- Advertencias y No verificados: la herramienta no puede garantizar su existencia. El ser humano es quién debe comprobar si el error existe realmente y solucionarlo posteriormente. Por ejemplo, contrastes de color de fondo y color de texto poco distinguible.
La revisión manual permitió comprobar el cumplimiento del: i) Criterio 1.3.2, indica que la secuencia en que se presenta el contenido no afecte su significado (Fig. 8); ii) Criterio 1.4.3., colores y contraste del sitio Web, donde se especifica un contraste mínimo en el texto (Fig. 9); iii) Criterio de éxito 1.4.4., posibilitando aumentar y disminuir el tamaño de de los textos desde las opciones situadas en la parte superior derecha del sitio $\mathrm{y}$ beneficiar a personas con deficiencia visual (Fig. 10).

TABLA 1. RESULTADOS DE LA EVALUACIÓN EN EL SITIO BANCARIO ${ }^{\circ} 1$

\begin{tabular}{|c|c|c|c|}
\hline Denominación de la Pagina & Problemas automáticos & Advertencias & No verificados \\
\hline \multirow{4}{*}{ Página 1} & \multirow{4}{*}{ Se carece de problemas de carácter automático } & Perceptible: 14 & Perceptible: 4 \\
\hline & & Operable: 17 & Operable: 7 \\
\hline & & Comprensible 6 & Comprensible 5 \\
\hline & & Robusto: 157 & Robusto: 1 \\
\hline \multirow{4}{*}{ Página 2} & \multirow{4}{*}{ Se carece de problemas de carácter automático } & Perceptible: 14 & Perceptible: 4 \\
\hline & & Operable: 17 & Operable: 7 \\
\hline & & Comprensible 6 & Comprensible 5 \\
\hline & & Robusto: 157 & Robusto: 1 \\
\hline \multirow{4}{*}{ Página 3} & \multirow{4}{*}{ Se carece de problemas de carácter automático } & Perceptible: 18 & Perceptible: 4 \\
\hline & & Operable: 17 & Operable: 7 \\
\hline & & Comprensible 6 & Comprensible 5 \\
\hline & & Robusto: 157 & Robusto: 1 \\
\hline \multirow{4}{*}{ Página 4} & \multirow{4}{*}{ Se carece de problemas de carácter automático } & Perceptible: 19 & Perceptible: 4 \\
\hline & & Operable: 17 & Operable: 7 \\
\hline & & Comprensible 6 & Comprensible 5 \\
\hline & & Robusto: 157 & Robusto: 1 \\
\hline \multirow{4}{*}{ Página 5} & \multirow{4}{*}{ Se carece de problemas de carácter automático } & Perceptible: 14 & Perceptible: 4 \\
\hline & & Operable: 17 & Operable: 7 \\
\hline & & Comprensible: 6 & Comprensible: 5 \\
\hline & & Robusto: 157 & Robusto: 1 \\
\hline \multirow{4}{*}{ Página 6} & \multirow{4}{*}{ Se carece de problemas de carácter automático } & Perceptible: 16 & Perceptible: 4 \\
\hline & & Operable: 17 & Operable: 7 \\
\hline & & Comprensible: 6 & Comprensible: 5 \\
\hline & & Robusto: 157 & Robusto: 1 \\
\hline \multirow{4}{*}{ Página 7} & \multirow{4}{*}{ Se carece de problemas de carácter automático } & Perceptible: 15 & Perceptible: 4 \\
\hline & & Operable: 23 & Operable: 7 \\
\hline & & Comprensible: 12 & Comprensible: 5 \\
\hline & & Robusto: 157 & Robusto: 1 \\
\hline
\end{tabular}

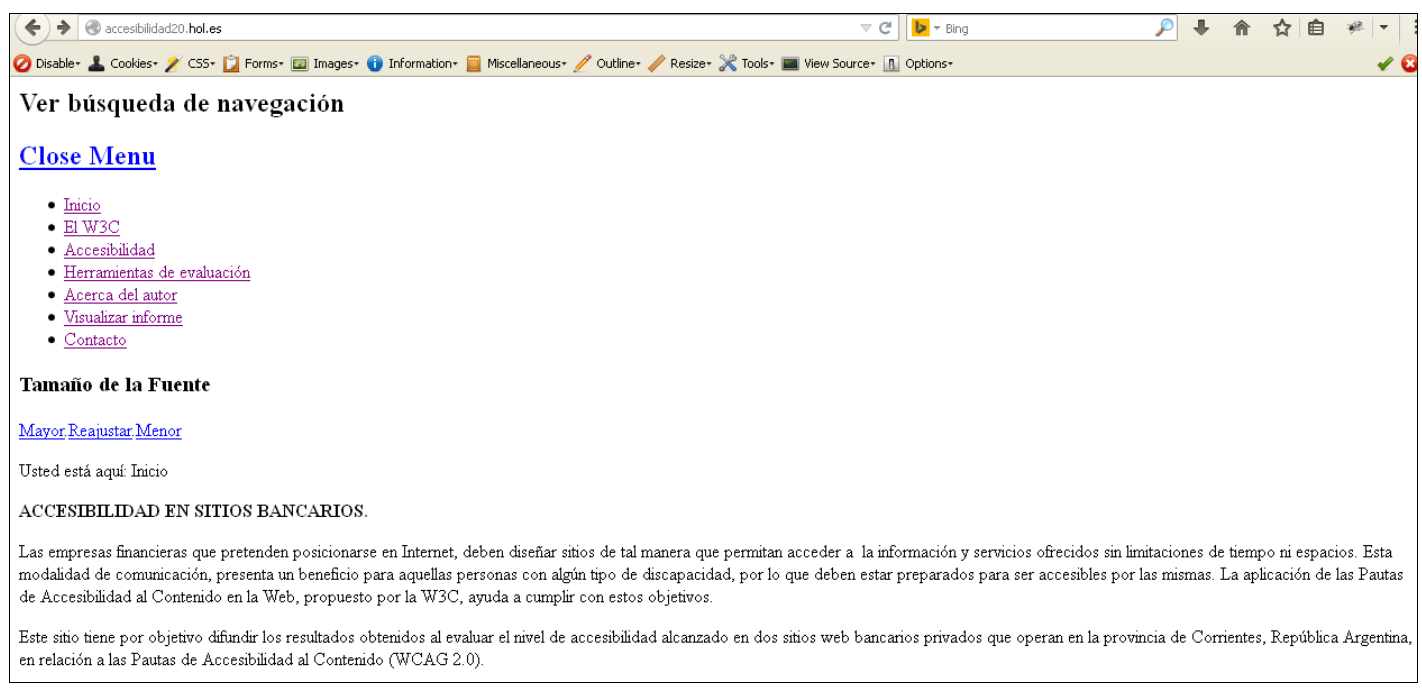

Fig. 81. Utilización CSS para controlar la presentación del texto. 


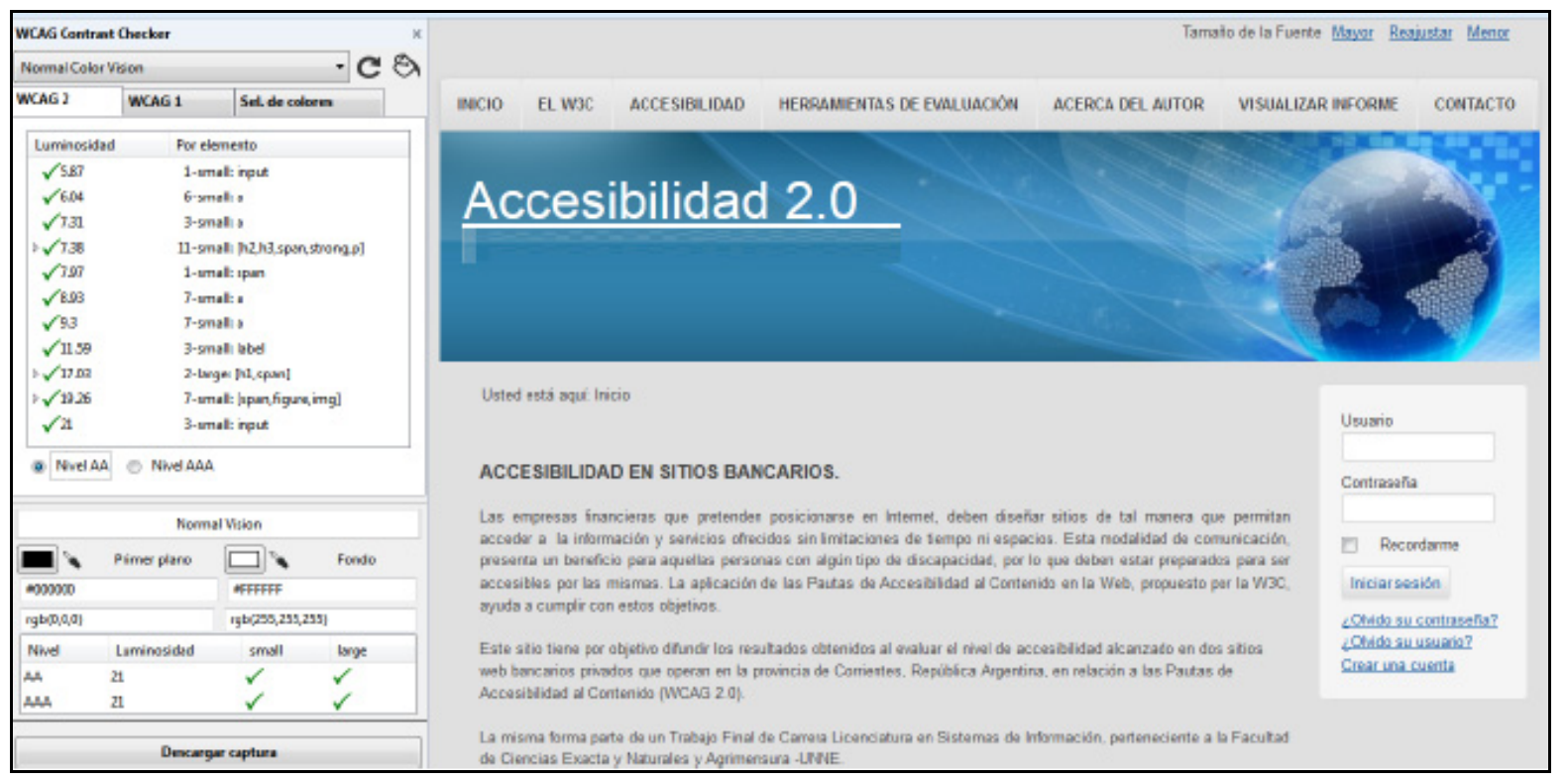

Fig. 9. Validación del contraste del sitio.

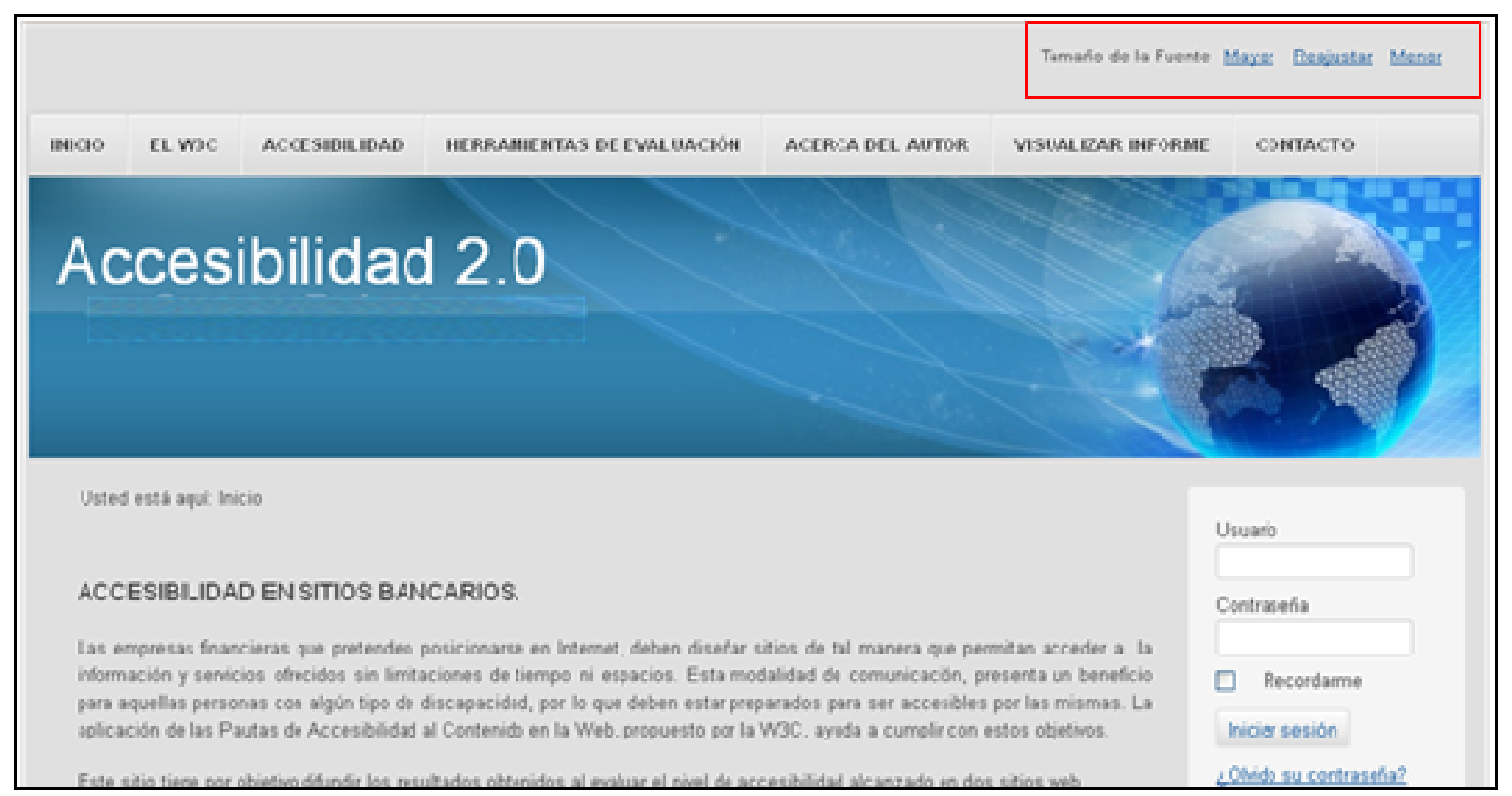

Fig. 102. Redimensión del tamaño del texto.

\section{CONCLUSIONES}

La evolución de las TIC, sus métodos y herramientas ha modificado el modo de almacenar, procesar y difundir información al tiempo que han requerido la implementación de estándares para asegurar el acceso a todos los e-ciudadanos sin distinguir capacidades visuales, físicas, cognitivas, de actualización en las herramientas de la web.

De la investigación documental bibliográfica abordada se puede afirmar que aun cuando la accesibilidad en Web ha sido ampliamente analizada y estudiada, se carece de un mayor número de sitios Web que la apliquen. Por lo expuesto lograr la accesibilidad en sitios Web beneficiaría a todos los usuarios, y mejoraría la aceptación de las TIC emergente en los inmigrantes digitales.

Como futuros trabajos se prevé continuar con la mejora sustancial de las plantillas por defecto proporcionadas por los CMS de libre distribución y aquellos productos que cumplan con los criterios de accesibilidad disponerlos a la comunidad de usuarios.

\section{REFERENCIAS}

[1] Oficina Española. Word Wide Web-Oficina Española-Guía Breve de Accesibilidad Web. [En línea]. Disponible en: http://www.w3c.es/divulgacion/guiasbreves/accesibilidad.[Acced ido: 29-mar-2014].

[2] ISO/IEC 40500:2012. Information technology - W3C Web Content Accessibility Guidelines (WCAG) 2.0. [En línea]. Disponible

http://www.iso.org/iso/iso_catalogue/catalogue_tc/catalogue_det ail.htm?csnumber=58625. [Accedido: 3-ago-2014].

[3] B. V. Bustos Torres, A. E. Martín y A. Cechich, "Extendiendo MVC para diseñar interfaces de usuario accesibiles", presentado en XIV Congreso Argentino de Ciencias de la Computación, 2008.

[4] Web Content Accessibility. Guidelines (WCAG) 2.0. [En línea]. Disponible en: http://www.w3.org/TR/WCAG20. [Accedido: 26jun-2014]. 
[5] S. Mariño, M. Godoy, P. Alfonzo, J. Acevedo, L. Gómez Solís y A. Fernández Vázquez, "Accesibilidad en la definición de requerimientos no funcionales. Revisión de herramientas", Multiciencias, vol 12, n 3, pp. 305-312, 2012

[6] TAW. Validador de accesibilidad. [En línea]. Disponible en: http://www.tawdis.net. [Accedido: 20-ago-2014].

[7] Web Developer Extension. [En línea]. Disponible en: https://addons.mozilla.org/es/firefox/addon/web-developer. [Accedido: 20-ago-2014].

[8] I. Sommerville, "Ingeniería del Software".7 $7^{a}$ Edición. Ed. Pearson, 2005.

[9] IEEE STD 1219:1993. Standard for Software Maintenance.IEEE Computer Society Press. USA, 1993.

[10] A. Gomez Codutti, S. I. Mariño, P. Alfonzo, M. V. Godoy, "Mantenimiento Perfectivo en un Gestor de Contenidos. Una Experiencia para la Especificación de Consultas Dinámicas". Revista Latinoamericana de Ingeniería de Software, 2(5): 310312, ISSN 2314-2642

[11] Joomla. [En línea]. Disponible en: http://www.joomla.org/.[Accedido: 20-jun-2014].

[12] Drupal. [En línea]. Disponible en: https://drupal.org/.[Accedido: 20-jun-2014].

[13] Moodle. [En línea]. Disponible en: https://moodle.org/?lang=es. [Accedido: 20-jun-2014].

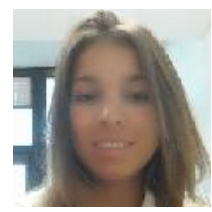

Daiana E. Casaro. Lic. en Sistemas de Información. Graduada de la Facultad de Ciencias Exactas y Naturales y Agrimensura Universidad Nacional del Nordeste (UNNE).

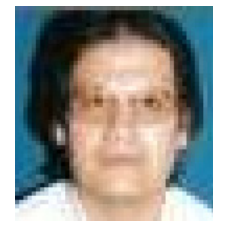

Pedro L. Alfonzo. Docente-Investigador. Experto en Estadística y Computación (Facultad de Ciencias Exactas y Naturales y Agrimensura UNNE). Especialista en Ingeniería de Software (Universidad Nacional de la Plata). Magíster en Ingeniería de Software (Universidad Nacional de La Plata).

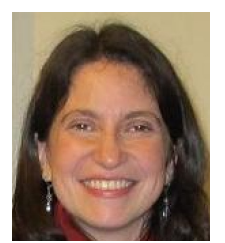

Sonia I. Mariño. Docente-Investigadora, Profesora Titular, Dedicación Exclusiva, del Departamento de Informática de la Facultad de Ciencias Exactas de la Universidad Nacional del Nordeste. Licenciada en Sistemas. Es Magíster en Informática y Computación. (UNNE - Universidad de Cantabria - España)., Magíster en Epistemología y Metodología de la Investigación Científica (Facultad de Humanidades - UNNE). Cursa el Doctorado en "Ciencias Cognitivas", Facultad de Humanidades (UNNE).

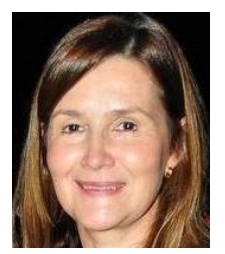

María V. Godoy. Docente-Investigadora, Profesora Titular, Dedicación Exclusiva, del Departamento de Informática de la Facultad de Ciencias Exactas de la Universidad Nacional del Nordeste. Experta en Estadística y Computación, y Licenciada en Sistemas. Es Magíster en Informática y Computación. (UNNE - Universidad de Cantabria - España). Cursa el Doctorado en "Ciencias Cognitivas", Facultad de Humanidades (UNNE). 